\title{
Hyers-Ulam stability of elliptic Möbius difference equation
}

\author{
Young Woo Nam \\ Mathematics Section, College of Science and Technology, Hongik University, \\ 339-701 Sejong, Korea
}

\begin{abstract}
The linear fractional map $f(z)=\frac{a z+b}{c z+d}$ on the Riemann sphere with complex coefficients $a d-b c \neq 0$ is called Möbius map. If $f$ satisfies $a d-b c=1$ and $-2<a+d<2$, then $f$ is called elliptic Möbius map. Let $\left\{b_{n}\right\}_{n \in \mathbb{N}_{0}}$ be the solution of the elliptic Möbius difference equation $b_{n+1}=f\left(b_{n}\right)$ for every $n \in \mathbb{N}_{0}$. Then the sequence $\left\{b_{n}\right\}_{n \in \mathbb{N}_{0}}$ has no Hyers-Ulam stability.
\end{abstract}

\section{Introduction}

The first order difference equation is the solution of $b_{n+1}=F\left(n, b_{n}\right)$ for $n \in \mathbb{N}_{0}$ with the initial point $b_{0}$. For the introductory method and examples see [3]. An interesting non-linear difference equation is the rational difference equation. For instance, Pielou logistic difference equation [7] or BevertonHolt equation [2, 9] are first order rational difference equation as a model for population dynamics with constraint. These equations are understood as the iteration of a kind of Möbius transformation on the real line. In this paper, we investigate the Hyers-Ulam stability of another kind of Möbius transformation which does not appear in population dynamics and extend the result to the complex plane.

Hyers-Ulam stability raised from Ulam's question [10] about the stability of approximate homomorphism between metric groups. The first answer to this question was given by Hyers [4] for Cauchy additive equation in Banach 
space. Later, the theory of Hyers-Ulam stability is developed in the area of functional equation and differential equation by many authors. The theory of Hyers-Ulam stability for difference equation appears in relatively recent decades and is mainly searched for linear difference equations, for example, see [5, 6, 8, 11]. Denote the set of natural numbers by $\mathbb{N}$ and denote the set $\mathbb{N} \cup\{\infty\}$ by $\mathbb{N}_{0}$. The set of real numbers and complex numbers by $\mathbb{R}$ and $\mathbb{C}$ respectively. Denote the unit circle by $\mathbb{S}^{1}$.

Suppose that the complex valued sequence $\left\{a_{n}\right\}_{n \in \mathbb{N}}$ satisfies the inequality

$$
\left|a_{n+1}-F\left(n, a_{n}\right)\right| \leq \varepsilon
$$

for a $\varepsilon>0$ and for all $n \in \mathbb{N}_{0}$, where $|\cdot|$ is the absolute value of complex number. If there exists a sequence $\left\{b_{n}\right\}_{n \in \mathbb{N}}$ which satisfies that

$$
b_{n+1}=F\left(n, b_{n}\right)
$$

for each $n \in \mathbb{N}_{0}$ and $\left|a_{n}-b_{n}\right| \leq G(\varepsilon)$ for all $n \in \mathbb{N}_{0}$, where the positive number $G(\varepsilon) \rightarrow 0$ as $\varepsilon \rightarrow 0$. Then we say that the difference equation (1.1) has Hyers-Ulam stability.

\section{Classification of Möbius transformation}

Denote the Riemann sphere by $\hat{\mathbb{C}}$, which is the one point compactification of the complex plane, namely, $\mathbb{C} \cup\{\infty\}$. Similarly, we define the extended real line as $\mathbb{R} \cup\{\infty\}$ and denote it by $\hat{\mathbb{R}}$. Möbius transformation (or Möbius map) is the linear fractional map defined on $\hat{\mathbb{C}}$ as follows

$$
g(z)=\frac{a z+b}{c z+d}
$$

where $a, b, c$ and $d$ are complex numbers and $a d-b c \neq 0$. Define $g\left(-\frac{d}{c}\right)=\infty$ and $g(\infty)=\frac{a}{c}$. If $c=0$, then $g$ is the linear function. Thus we assume that $c \neq 0$ throughout this paper. The Möbius map which preserves $\hat{\mathbb{R}}$ is called the real Möbius map. A Möbius map is real if and only if the coefficients of the map $a, b, c$ and $d$ are real numbers.

The Möbius map has two fixed points counting with multiplicity. Denote these points by $\alpha$ and $\beta$. The real Möbius maps are classified to the three different cases using fixed points. 
- If $\alpha$ and $\beta$ are real distinct numbers, the map is called real hyperbolic Möbius map,

- If $\alpha=\beta$, then the map is called real parabolic Möbius map, and

- If $\alpha$ and $\beta$ are two distinct non-real complex numbers, then the map is called real elliptic Möbius map.

Möbius map $x \mapsto \frac{a x+b}{c x+d}$ is the same as $x \mapsto \frac{p a x+p b}{p c x+p d}$ for all numbers $p \neq 0$. Thus we may assume that $a d-b c=1$ when we choose $p=\sqrt{a d-b c}$. Moreover, Möbius map has the matrix representation $\left(\begin{array}{ll}a & b \\ c & d\end{array}\right)$ under the condition $a d-$ $b c=1$. Denote the matrix representation of the Möbius map $g$, by also $g$ and its trace by $\operatorname{tr}(g)$, which means $a+d$. In the complex analysis or hyperbolic geometry, Möbius maps with complex coefficients can be classified similarly with different method. For instance, see [1]. Möbius transformation in (1.2) (with real or complex coefficients) for $a d-b c=1$ is classified as follows

- If $\operatorname{tr}(g) \in \mathbb{R} \backslash[-2,2]$, then $g$ is called hyperbolic,

- If $\operatorname{tr}(g)= \pm 2$, then $g$ is called parabolic,

- If $\operatorname{tr}(g) \in(-2,2)$, then $g$ is called elliptic and

- If $\operatorname{tr}(g) \in \mathbb{C} \backslash \mathbb{R}$, then $g$ is called purely loxodromic.

The real Möbius maps are also classified by the above notions. In this paper, we investigate Hyers-Ulam stability of elliptic Möbius transformations. Other cases would appear in the forthcoming papers.

\section{No Hyers-Ulam stability with dense subset}

In this section we prove non-stability in the sense of Hyers-Ulam, which is not only for the elliptic Möbius transformation but also for any function satisfying the assumption of the following Theorem 2.1.

Theorem 2.1. Let $\left\{b_{n}\right\}_{n \in \mathbb{N}_{0}}$ be the sequence in $\mathbb{R}$ satisfying $b_{n+1}=F\left(b_{n}\right)$ with a map $F$ for $n \in \mathbb{N}_{0}$. Suppose that there exists a dense subset $A$ of $\mathbb{R}$ such that if $b_{0} \in A$, then the sequence $\left\{b_{n}\right\}_{n \in \mathbb{N}_{0}}$ is dense in $\mathbb{R}$. Suppose also that $\left\{b_{n}\right\}_{n \in \mathbb{N}_{0}}$ has no periodic point. Then the sequence $\left\{b_{n}\right\}_{n \in \mathbb{N}_{0}}$ has no Hyers-Ulam stability. 
Proof. For any $a_{0} \in \mathbb{R}$ and $\varepsilon>0$, choose the sequence $\left\{a_{n}\right\}_{n \in \mathbb{N}_{0}}$ as follows

1. $a_{0} \in \mathbb{R}$ is arbitrary,

2. $a_{1}$ satisfies that $\left|F\left(a_{0}\right)-a_{1}\right| \leq \varepsilon$ and $a_{1} \in A$, that is, the sequence $\left\{F^{n}\left(a_{1}\right)\right\}_{n \in \mathbb{N}_{0}}$ is dense in $\mathbb{R}$.

Let $n_{k}$ for $k \geq 1$ be the positive numbers $n_{1}<n_{2}<\cdots<n_{k}<\cdots$ such that $F^{n_{k}}\left(a_{1}\right)$ are points in the ball of which center is $a_{1}$ and diameter is $\varepsilon$.

3. $a_{n+1}=F^{n}\left(a_{1}\right)$ for $n=0,1,2, \ldots, n_{1}-1$,

4. $a_{n_{1}+1}=a_{1}$ and $a_{k}=a_{k+n_{1}+1}$ for every $k \in \mathbb{N}$.

Then the sequence $\left\{a_{n}\right\}_{n \in \mathbb{N}_{0}}$ satisfies that $\left|a_{n+1}-F\left(a_{n}\right)\right| \leq \varepsilon$ for all $n \in \mathbb{N}_{0}$. Moreover, since the sequence $\left\{a_{n}\right\}_{n \in \mathbb{N}}$ is periodic, $\left\{a_{n}\right\}_{n \in \mathbb{N}_{0}}$ is the finite set and it is bounded. However, the fact that the sequence $\left\{b_{n}\right\}_{n \in \mathbb{N}_{0}}$ is dense in $\mathbb{R}$ implies that $\left|a_{n}-b_{n}\right|$ is unbounded for $n \in \mathbb{N}$. Hence, the sequence $\left\{b_{n}\right\}_{n \in \mathbb{N}_{0}}$ does not have Hyers-Ulam stability.

Remark 2.2. Theorem 2.1 can be generalized to any metric space only if the definition of Hyers-Ulam stability is modified suitably. For instance, if $F$ is the map from the metric space $X$ to itself and $|\cdot|$ is changed to the distance $\operatorname{dist}(\cdot, \cdot)$ from the metric on $X$, then we can define Hyers-Ulam stability on the metric space and Theorem 2.1 is applied to it. For example, the unit circle $\mathbb{S}^{1}$ is the metric space of which distance between two points defined from the minimal arc length connecting these two points. Then Hyers-Ulam stability on $\mathbb{S}^{1}$ can be defined.

\section{Real elliptic Möbius transformation}

Lemma 3.1. Let $g(x)=\frac{a x+b}{c x+d}$ be the linear fractional map where $a, b, c$ and $d$ are real numbers, $c \neq 0$ and $a d-b c=1$. Then $g$ has fixed points which are non-real complex numbers if and only if $g$ is real elliptic Möbius map, that $i s,-2<a+d<2$.

Proof. The equation $g(x)=x$ implies that

$$
x=\frac{a-d \pm \sqrt{(a+d)^{2}-4}}{2 c} .
$$

Then the fixed points are non-real complex numbers if and only if $-2<$ $a+d<2$. 
Lemma 3.2. Let $g$ be the map defined on $\hat{\mathbb{C}}$ in Lemma 3.1 and two complex numbers $\alpha$ and its complex conjugate $\bar{\alpha}$ be the fixed points of $g$. Let $h$ be the map defined as $h(x)=\frac{x-\alpha}{x-\bar{\alpha}}$. If $g$ is the elliptic Möbius map, that is, $-2<a+d<2$, then

$$
h \circ g \circ h^{-1}(x)=\frac{x}{(c \alpha+d)^{2}} .
$$

for $x \in \hat{\mathbb{C}}$. Moreover, $\left|g^{\prime}(\alpha)\right|=|c \alpha+d|=1$.

Proof. The map $h \circ g \circ h^{-1}$ has the fixed points 0 and $\infty$. Since both $g$ and $h$ are linear fractional maps, so is $h \circ g \circ h^{-1}$. Then $h \circ g \circ h^{-1}(x)=k x$ for some $k \in \mathbb{C}$. The equation $h \circ g(x)=k h(x)$ implies that $h^{\prime}(g(x)) g^{\prime}(x)=k h^{\prime}(x)$. Thus

$$
h^{\prime}(g(\alpha)) g^{\prime}(\alpha)=h^{\prime}(\alpha) g^{\prime}(\alpha)=k h^{\prime}(\alpha)
$$

Then $k=g^{\prime}(\alpha)=\frac{1}{(c \alpha+d)^{2}}$. Moreover, $\left|g^{\prime}(\alpha)\right|=1$ if and only if $|c \alpha+d|=1$. Recall that both $\alpha$ and $\bar{\alpha}$ are roots of the equation, $c x^{2}-(a-d) x-b=0$. Then

$$
\begin{aligned}
|c \alpha+d|^{2} & =(c \alpha+d)(c \bar{\alpha}+d) \\
& =c^{2} \alpha \bar{\alpha}+c d(\alpha+\bar{\alpha})+d^{2} \\
& =c^{2}\left(-\frac{b}{c}\right)+c d \frac{a-d}{c}+d^{2} \\
& =-b c+a d-d^{2}+d^{2} \\
& =a d-b c \\
& =1
\end{aligned}
$$

Hence, $\left|g^{\prime}(\alpha)\right|=\frac{1}{|c \alpha+d|^{2}}=1$.

By Lemma 3.2, the map $h \circ g \circ h^{-1}$ is a rotation on $\mathbb{S}^{1}$. Since $h^{-1}$ is bijective from $\mathbb{S}^{1} \backslash\{1\}$ to $\mathbb{R}, x$ is a periodic point under $h \circ g \circ h^{-1}$ in $\mathbb{S}^{1} \backslash\{1\}$ with period $p$ if and only if $h^{-1}(x)$ is periodic in $\mathbb{R}$ with the same period. Recall that $h^{-1}(1)=\infty$. Thus when we investigate Hyers-Ulam stability of the sequence $\left\{b_{n}\right\}_{n \in \mathbb{N}_{0}}$ as the solution of the elliptic linear fractional map $g$, we have to choose carefully the initial point $b_{0} \in \mathbb{R}$ satisfying $g^{k}\left(b_{0}\right) \neq \infty$ for all $k \in \mathbb{N}$. 
Proposition 3.3. Let $g$ be the elliptic linear fractional map defined in Lemma 3.1 on $\hat{\mathbb{R}}$. Suppose that there exists $x \in \mathbb{R}$ such that $g^{k}(x) \neq x$ for all $k \in \mathbb{N}$. Then the sequence $\left\{g^{k}(x)\right\}_{k \in \mathbb{N}}$ is dense in $\mathbb{R}$ where $x \in \mathbb{R} \backslash\left\{g^{-k}(\infty)\right\}_{k \in \mathbb{N}}$.

Proof. Lemma 3.2 implies that $h \circ g \circ h^{-1}(x)=e^{i \theta} x$ for some $\theta \in \mathbb{R}$. If $\theta$ is a rational number $\frac{q}{p}$, then $h \circ g^{p} \circ h^{-1}(x)=x$ for all $x \in \mathbb{C}$. Thus $g^{p}(x)=x$ for all $x \in \mathbb{C}$. Then $\theta$ is an irrational number. Since $x \mapsto e^{i \theta} x$ is an irrational rotation on $\mathbb{S}^{1}$, the sequence $\left\{e^{i k \theta} x\right\}_{k \in \mathbb{N}}$ is dense in $\mathbb{S}^{1}$ for every $x \in \mathbb{S}^{1}$. Moreover, when $x=1$ is chosen, the set $\mathbb{S}^{1} \backslash\left\{e^{-i k \theta}\right\}_{k \in \mathbb{N}_{0}}$ is also a dense subset of $\mathbb{S}^{1}$.

The direct calculation implies that $h^{-1}(x)=\frac{\bar{\alpha} x-\alpha}{x-1}$ and then $h^{-1}(1)=\infty$. Choose two points $p$ and $p^{\prime}$ close enough to each other in $\mathbb{S}^{1} \backslash\left\{e^{-i k \theta}\right\}_{k \in \mathbb{N}_{0}}$. Then

$$
\begin{aligned}
\left|h^{-1}(p)-h^{-1}\left(p^{\prime}\right)\right| & =\left|\frac{\bar{\alpha} p-\alpha}{p-1}-\frac{\bar{\alpha} p^{\prime}-\alpha}{p^{\prime}-1}\right| \\
& =\left|\frac{(\bar{\alpha} p-\alpha)\left(p^{\prime}-1\right)-\left(\bar{\alpha} p^{\prime}-\alpha\right)(p-1)}{(p-1)\left(p^{\prime}-1\right)}\right| \\
& =\left|\frac{(\alpha-\bar{\alpha})\left(p-p^{\prime}\right)}{(p-1)\left(p^{\prime}-1\right)}\right| \\
& =\left|\frac{\alpha-\bar{\alpha}}{(p-1)\left(p^{\prime}-1\right)}\right| \cdot\left|p-p^{\prime}\right| .
\end{aligned}
$$

We choose the sequence $\left\{p_{n_{k}}\right\}_{k \in \mathbb{N}_{0}}$ for some $p_{0} \in \mathbb{S}^{1} \backslash\left\{e^{-i k \theta}\right\}_{k \in \mathbb{N}_{0}}$ which satisfies that $h \circ g^{n_{k}} \circ h^{-1}\left(p_{0}\right)=p_{n_{k}}$ and $p_{n_{k}} \rightarrow p$ as $k \rightarrow \infty$ for different numbers $n_{1}<n_{2}<\cdots<n_{k}<\cdots$. Since $h^{-1}$ is a bijection from $\mathbb{S}^{1} \backslash\{1\}$ to $\mathbb{R}$, by the equation (3.1) we obtain that

$$
\begin{aligned}
\left|h^{-1}(p)-g^{n_{k}} \circ h^{-1}\left(p_{0}\right)\right| & =\left|h^{-1}(p)-h^{-1}\left(p_{n_{k}}\right)\right| \\
& \leq\left(\left|\frac{\alpha-\bar{\alpha}}{(p-1)^{2}}\right|+\delta\right)\left|p-p_{n_{k}}\right|
\end{aligned}
$$

for some $\delta>0$. Then any point $h^{-1}(p)$ in $\mathbb{R}$ is an accumulation point in the sequence $\left\{g^{k}(x)\right\}_{k \in \mathbb{N}_{0}}$ where $x \in h^{-1}\left(\mathbb{S}^{1} \backslash\left\{e^{-i k \theta}\right\}_{k \in \mathbb{N}_{0}}\right)$, which is a dense subset of $\mathbb{R}$.

Theorem 2.1 and Proposition 3.3 imply that the real elliptic linear fractional map does not have Hyers-Ulam stability. 
Corollary 3.4. Let $g(x)=\frac{a x+b}{c x+d}$ be the linear fractional map on $\hat{\mathbb{R}}$ where $a, b, c$ and $d$ are real numbers, $c \neq 0$ and $a d-b c=1$. Suppose that $-2<$ $a+d<2$ and $h \circ g \circ h^{-1}$ is an irrational rotation on the unit circle where $h(x)=\frac{x-\alpha}{x-\bar{\alpha}}$. If the sequence $\left\{b_{n}\right\}_{n \in \mathbb{N}_{0}}$ in $\mathbb{R}$ is the solution of $b_{n+1}=g\left(b_{n}\right)$ for $n \in \mathbb{N}_{0}$, then either $g^{k}\left(b_{0}\right)=\infty$ for some $k \in \mathbb{N}$ or $\left\{b_{n}\right\}_{n \in \mathbb{N}_{0}}$ has no Hyers-Ulam stability .

\section{Extension of non stability to complex plane}

In this section, we extend no Hyers-Ulam stability of the real elliptic linear fractional map to the elliptic Möbius transformation with complex coefficients. Let $\ell$ be the straight line in the complex plane. Define the extended line as $\ell \cup\{\infty\}$ and denote it by $\hat{\ell}$. Interior of the circle $C$ in $\mathbb{C}$ means that the bounded region of the set $\mathbb{C} \backslash C$.

Lemma 4.1. Let $f(z)=\frac{a z+b}{c z+d}$ be the Möbius map with complex coefficients $a, b, c$ and $d$ for $c \neq 0$ and $a d-b c=1$. If $f$ is elliptic, that $i s,-2<a+d<2$, then there exists the unique extended line which is invariant under $g$ in $\hat{\mathbb{C}}$.

Proof. Let $\alpha$ and $\beta$ be the fixed points of $f$. In particular, the fixed points of $g$ are as follows

$$
\alpha=\frac{a-d+\sqrt{(a+d)^{2}-4}}{2 c}, \quad \beta=\frac{a-d-\sqrt{(a+d)^{2}-4}}{2 c}
$$

Denote the straight line, $\{z \in \mathbb{C}:|z-\alpha|=|z-\beta|\}$ by $\ell$ in $\mathbb{C}$ and denote the extended line $\ell \cup\{\infty\}$ by $\hat{\ell}$. We prove that $\hat{\ell}$ is the unique invariant extended line under $f$.

Claim: The points, $-\frac{d}{c}$ and $\frac{a}{c}$ are in $\hat{\ell}$.

$$
\alpha+\frac{d}{c}=\frac{a+d+\sqrt{(a+d)^{2}-4}}{2 c}, \quad \beta+\frac{d}{c}=\frac{a+d-\sqrt{(a+d)^{2}-4}}{2 c}
$$

The fact that $a+d$ is a real number and $\sqrt{(a+d)^{2}-4}$ is a purely imaginary number implies that

$$
\left|\alpha+\frac{d}{c}\right|=\left|\beta+\frac{d}{c}\right|=\frac{1}{|c|}
$$


Then $-\frac{d}{c}$ is in $\hat{\ell}$. By similar calculation, $\frac{a}{c}$ is also in $\hat{\ell}$. The proof the claim is complete.

For the invariance of $\hat{\ell}$, it suffice to show that if $z \in \ell \backslash\left\{-\frac{d}{c}\right\}$, then $f(z) \in \ell$. For any $z \in \ell \backslash\left\{-\frac{d}{c}\right\}$, we have that

$$
\begin{aligned}
f(z)-\alpha & =f(z)-f(\alpha) \\
& =\frac{a z+b}{c z+d}-\frac{a \alpha+b}{c \alpha+d}=\frac{(a d-b c)(z-\alpha)}{(c z+d)(c \alpha+d)}=\frac{z-\alpha}{(c z+d)(c \alpha+d)}
\end{aligned}
$$

By similar calculation, we obtain that

$$
f(z)-\beta=\frac{z-\beta}{(c z+d)(c \beta+d)} .
$$

The equation (4.1) in the claim implies that $|c \alpha+d|=|c \beta+d|$. The definition of $\ell$ implies that $c z+d \neq 0$ for $z \in \ell \backslash\left\{-\frac{d}{c}\right\}$ and $|z-\alpha|=|z-\beta|$. The equation $|f(z)-\alpha|=|f(z)-\beta|$ holds by comparing the equation (4.2) with (4.3). Hence, by this result and the above claim, the extended line $\hat{\ell}$ is invariant under $f$. There is the unique straight line which connects $-\frac{d}{c}$ and $\frac{a}{c}$ in $\mathbb{C}$. Hence, $\hat{\ell}$ is the unique invariant extended line in $\hat{\mathbb{C}}$ under $f$.

Remark 4.2. Define the map $h$ as $h(z)=\frac{z-\beta}{z-\alpha}$. Then by the straightforward calculation we obtain $h \circ f \circ h^{-1}(z)=f^{\prime}(\alpha) z$ and $\left|f^{\prime}(\alpha)\right|=1$. Let $C_{r}$ be the circle of radius $r>0$ of which center is the origin in $\mathbb{C}$. Observe that a fixed point of $f$ is contained in the interior of $h^{-1}\left(C_{r}\right)$ for all $r>0$. If $h \circ g \circ h^{-1}$ is an irrational rotation in $\mathbb{C}$, then every concentric circles $C_{r}$ are invariant under $h \circ f \circ h^{-1}$. Then $h^{-1}\left(C_{r}\right)$ is an invariant circle under $f$ for every $r>0$. However, since $h(\infty)=1$, the unique invariant extended line under $f$ is $h^{-1}\left(\mathbb{S}^{1}\right)$. Moreover, $h^{-1}\left(C_{1 / r}\right)$ is the reflected image of $h^{-1}\left(C_{r}\right)$ to the invariant extended line.

Due to the existence of the extended line which is invariant under $g$, no Hyers-Ulam stability of the sequence $\left\{g^{n}(z)\right\}_{n \in \mathbb{N}_{0}}$ of the real elliptic linear fractional map is extendible to that of the elliptic Möbius transformation.

Proposition 4.3. Let $f$ be the elliptic Möbius transformation on $\hat{\mathbb{C}}$ and $\hat{\ell}$ be the extended line invariant under $f$. Suppose that there exists $z \in \hat{\ell}$ such that $f^{k}(z) \neq z$ for all $k \in \mathbb{N}$. Then the sequence $\left\{f^{k}(z)\right\}_{k \in \mathbb{N}}$ is dense in $\ell=\hat{\ell} \backslash\{\infty\}$ where $z \in \ell \backslash\left\{f^{-k}(\infty)\right\}_{k \in \mathbb{N}}$. 
Proof. Lemma 4.1 implies that there exists the invariant extended line under $f$. In the proof of Proposition $[3.3$, replace $\bar{\alpha}$ by $\beta$ and apply the proof of Proposition 3.3. Then the similar calculation completes the proof.

Then Proposition 4.3 and Theorem 2.1 implies that no Hyers-Ulam stability of $\left\{f^{n}(z)\right\}_{n \in \mathbb{N}_{0}}$ where $f$ is the elliptic Möbius map for $z \in \mathbb{C}$.

Corollary 4.4. Let $f(z)=\frac{a z+b}{c z+d}$ be the Möbius map on $\hat{\mathbb{C}}$ where $c \neq 0$ and $a d-b c=1$. If $f$ is elliptic, that is, $-2<a+d<2$, then the sequence $\left\{b_{n}\right\}_{n \in \mathbb{N}_{0}}$ satisfying $b_{n+1}=f\left(b_{n}\right)$ for $n \in \mathbb{N}_{0}$ has no Hyers-Ulam stability on $\mathbb{C}$.

Proof. The sequence $\left\{a_{n}\right\}_{n \in \mathbb{N}_{0}}$ is in the invariant extended line $\hat{\ell}$ under $f$ satisfying

$$
\left|a_{n+1}-f\left(a_{n}\right)\right| \leq \varepsilon
$$

for $n \in \mathbb{N}_{0}$. Choose $\left\{a_{n}\right\}_{n \in \mathbb{N}_{0}}$ is the finite sequence which satisfies the properties in the proof of Theorem 2.1 in $\ell$. Then the similar proof in Section 3 implies that any sequence $\left\{b_{n}\right\}_{n \in \mathbb{N}_{0}}$ satisfying $b_{n+1}=f\left(b_{n}\right)$ for every $n \in \mathbb{N}_{0}$ in $\ell$ has no Hyers-Ulam stability.

Let $h$ be the map $h(z)=\frac{z-\alpha}{z-\beta}$. Observe that $h \circ f \circ h^{-1}$ is an irrational rotation on $\mathbb{C}$. If $\left\{b_{n}\right\}_{n \in \mathbb{N}_{0}}$ is contained in $\mathbb{C} \backslash \hat{\ell}$, then $\left\{b_{n}\right\}_{n \in \mathbb{N}_{0}}$ is contained in the circle disjoint from $\ell$ and this circle is $h^{-1}\left(C_{r}\right)$ for some $r>0$. Observe that if $r=0$, then $C_{r}=\{\alpha\}$ and if $r=1$, then $C_{r}=\hat{\ell}$. Since $h \circ f \circ h^{-1}$ is an irrational rotation, $\left\{f^{n}\left(h^{-1}(x)\right)\right\}_{n \in \mathbb{N}_{0}}$ is the dense subset of $h^{-1}\left(C_{r}\right)$. We may assume that $0<r<1$ and the fixed point $\alpha$ is contained in the interior of $C_{r}$ by Remark 4.2. Denote the (minimal) distance between the point $\alpha$ and the line $\ell$ by $L$. Then the density of $\left\{b_{n}\right\}_{n \in \mathbb{N}_{0}}$ on the circle $C_{r}$ and the finiteness of $\left\{a_{n}\right\}_{n \in \mathbb{N}_{0}}$ imply that $\left|a_{n}-b_{n}\right| \geq L$ for infinitely many $n \in \mathbb{N}$ for all $\varepsilon<L$. Hence, $\left\{b_{n}\right\}_{n \in \mathbb{N}_{0}}$ has no Hyers-Ulam stability.

\section{Non stability of periodic sequence}

Let the sequence $\left\{b_{n}\right\}_{n \in \mathbb{N}_{0}}$ satisfying $b_{n+p}=b_{n}$ for every $n \in \mathbb{N}_{0}$ for some $p \in \mathbb{N}$ be periodic sequence. The least positive number $p$ satisfying the above equation is called the peroid of sequence. If $p=1$, then it is called constant sequence. 
Lemma 5.1. Let the sequence $\left\{b_{n}\right\}_{n \in \mathbb{N}_{0}}$ in $\mathbb{R}$ be a periodic sequence with period $p$. Then $\left\{b_{n}\right\}_{n \in \mathbb{N}_{0}}$ has no Hyers-Ulam stability.

Proof. Any periodic sequence has constant subsequence. For example, let $\left\{c_{n}\right\}_{n \in \mathbb{N}_{0}}$ be the sequence satisfying $c_{n}=b_{p n}$ for every $n \in \mathbb{N}_{0}$. Thus $\left\{c_{n}\right\}_{n \in \mathbb{N}_{0}}$ is the constant sequence $\left\{c_{0}\right\}_{n \in \mathbb{N}_{0}}$. It suffice to show that the constant sequence has no Hyers-Ulam stability. For any small enough $\varepsilon>0$, define the sequence $\left\{d_{n}\right\}_{n \in \mathbb{N}_{0}}$ as follows

1. $d_{0}$ is arbitrary and

2. $d_{n}=d_{0}+n \varepsilon$ for $n \in \mathbb{N}$

Then $\left|d_{n+1}-d_{n}\right| \leq \varepsilon$ for all $n \in \mathbb{N}_{0}$. However, for any constant sequence $\left\{c_{0}\right\}_{n \in \mathbb{N}_{0}}$ satisfying $\left|c_{0}-d_{0}\right| \leq \varepsilon$ such that

$$
\left|c_{k}-d_{k}\right|=\left|c_{0}-d_{k}\right|=\left|c_{0}-d_{0}-n \varepsilon\right| \geq-\left|c_{0}-d_{0}\right|+n \varepsilon \geq(n-1) \varepsilon
$$

for all $n \geq 2$. Since $\left|c_{k}-d_{k}\right|$ is unbounded for $n \in \mathbb{N}$, the constant sequence $\left\{c_{n}\right\}_{n \in \mathbb{N}_{0}}$ has no Hyers-Ulam stability. Hence, the periodic sequence $\left\{b_{n}\right\}_{n \in \mathbb{N}_{0}}$ has no Hyers-Ulam stability either.

Example 5.2. There are non-linear Möbius transformations, of which finitely many composition is the identity map. For example, see the following maps

$$
p(z)=\frac{\sqrt{3} z-2}{2 z-\sqrt{3}}, \quad q(z)=\frac{-1}{z-\sqrt{3}}, \quad r(z)=\frac{-z-1}{z} .
$$

Thus $p \circ p(z)=p^{2}(z)=z$ for all $z \in \mathbb{C}$ and the trace of $p$ is that $\operatorname{tr}(p)=$ $\sqrt{3}+(-\sqrt{3})=0$. The map $q$ satisfies that $q^{3}=p$ by the direct calculation and then $q^{6}(z)=z$ for all $z \in \mathbb{C}$. The trace of $q$ is that $\operatorname{tr}(q)=0+(-\sqrt{3})=-\sqrt{3}$. Finally, $r^{3}(z)=z$ for all $z \in \mathbb{C}$ and $\operatorname{tr}(r)=-1+0=-1$. All of the traces of $p, q$ and $r$ are between -2 and 2 .

Remark 4.2 implies that $h \circ g \circ h^{-1}(x)=e^{i \theta} x$ for every elliptic Möbius map $g$. If $\theta=\frac{q}{p}$, then $g^{p}(x)=x$ for all $x \in \mathbb{C}$. Thus the sequence $\left\{g^{n}(x)\right\}_{n \in \mathbb{N}_{0}}$ is periodic. Then Corollary 3.4 and Lemma 5.1 implies the following theorem. 
Theorem 5.3. Let $g(x)=\frac{a x+b}{c x+d}$ be the linear fractional map on $\hat{\mathbb{R}}$ for $c \neq 0$, $a d-b c=1$. Supposet that $g$ is the elliptic linear fractional map, that is, $-2<a+d<2$. Then the sequence $\left\{b_{n}\right\}_{n \in \mathbb{N}_{0}}$ in $\mathbb{R}$ satisfying $b_{n+1}=g\left(b_{n}\right)$ for $n \in \mathbb{N}_{0}$ either satisfies that $g^{k}\left(b_{0}\right)=\infty$ for some $k \in \mathbb{N}$ or it has no Hyers-Ulam stabiliy.

If the sequence $\left\{f^{n}(z)\right\}_{n \in \mathbb{N}_{0}}$ is periodic, then Corollary 4.4 and Lemma 5.1 implies the following theorem.

Theorem 5.4. Let $f(z)=\frac{a z+b}{c z+d}$ be the Möbius map on $\hat{\mathbb{C}}$ for $a d-b c=1$, $c \neq 0$. Suppose that $f$ is the elliptic Möbius map, that is, $-2<a+d<2$. Then the sequence $\left\{b_{n}\right\}_{n \in \mathbb{N}_{0}}$ in $\mathbb{C}$ satisfying $b_{n+1}=f\left(b_{n}\right)$ for $n \in \mathbb{N}_{0}$ either satisfies that $g^{k}\left(b_{0}\right)=\infty$ for some $k \in \mathbb{N}$ or it has no Hyers-Ulam stabiliy.

\section{References}

[1] Beardon, A F: The geometry of discrete groups. Springer-Verlag, Graduate Texts in Mathematics 91 (1983)

[2] Bohner, M, Warth, H: The Beverton-Holt dynamic equation. Applicable Analysis 86, 1007-1015 (2007)

[3] Elaydi, S N: An Introduction to Difference Equations. Springer (2005)

[4] Hyers, D H: On the stability of the linear functional equation. Proc. Natl. Acad. Sci. USA 27, 222-224 (1941)

[5] Jung, S-M: Hyers-Ulam stability of the first-order matrix difference equations. Adv. Difference Equ. no. 170 (2015)

[6] Jung, S-M, Nam, Y W: On the Hyers-Ulam stability of the first order difference equation. J. Function Spaces, Article ID 6078298 (2016)

[7] Pielou, E C: Population and community ecology. Gordon and Breach, Science publishers (1974)

[8] Popa, D: Hyers-Ulam-Rassias stability of a linear recurrence. J. Math. Anal. Appl. 309, 591-597 (2015)

[9] Sen, M De la: The generalized Beverton-Holt equation and the control of populations. Applied Mathematical Modeling 32, 2312-2328 (2008) 
[10] Ulam, S M: A Collection of Mathematical Problems. Interscience Publ., New York (1960)

[11] Xu, B, Brzdęk, J: Hyers-Ulam stability of a system of first order linear recurrences with constant coefficients. Discrete Dyn. Nat. Soc. Article ID 269356 (2015) 